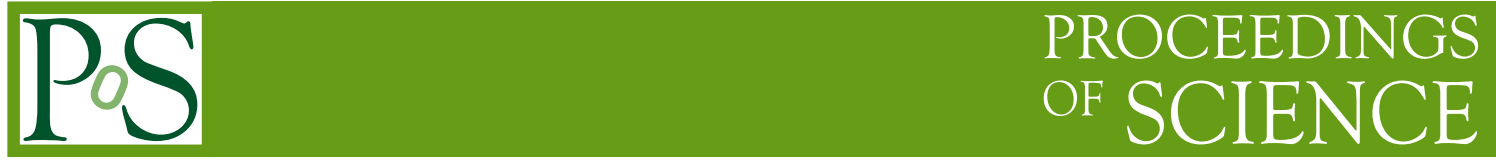

\title{
Precision measurement of the top quark mass in lepton+jets channels
}

\author{
Jochen Cammin*i \\ University of Rochester, NY (USA) \\ E-mail: camminefnal.gov
}

\begin{abstract}
The measurement of the top-quark mass in lepton+jets channels provides the most precise determination of the top-quark mass from Tevatron Run I data. In the Run II phase, the CDF and $\mathrm{D} \emptyset$ experiments have already collected more than $1 \mathrm{fb}^{-1}$ of data in the period 2002-2005. The preliminary analyses presented here use a subset of up to $320 \mathrm{pb}^{-1}$. With these statistics and an in-situ calibration of the jet energies, a single analysis now surpasses the precision of the previous world average.
\end{abstract}

International Workshop on Top Quark Physics

January 12-15, 2006

Coimbra, Portugal

\footnotetext{
* Speaker.

${ }^{\dagger}$ On behalf of the CDF and DØ collaborations.
} 


\section{Introduction}

The production of top-quarks has been measured at the Tevatron in the pair production process $\mathrm{q} \overline{\mathrm{q}} / \mathrm{gg} \rightarrow \mathrm{t} \overline{\mathrm{t}}$. Since the top quark decays almost exclusively via $\mathrm{t} \rightarrow \mathrm{bW}$, the final state is classified through the decay mode of the $\mathrm{W}$ boson. For $\mathrm{t} \overline{\mathrm{t}}$ events, this leads to the dilepton channels $\mathrm{t} \overline{\mathrm{t}} \rightarrow$ $\ell v \mathrm{~b} \ell v \mathrm{~b}$, the lepton+jets channels $\mathrm{t} \overline{\mathrm{t}} \rightarrow \ell v \mathrm{bjjb}$, and the fully-hadronic channel $\mathrm{t} \overline{\mathrm{t}} \rightarrow \mathrm{jjb} \mathrm{jj} b$. The dilepton channels have the cleanest signature due to large missing energy from two neutrinos and two isolated leptons. However, the branching ratio is only $5 \%$ (if one considers only electrons and muons as leptons). The all-hadronic channel has a large branching ratio of about $45 \%$, but suffers from very large backgrounds. The best compromise between signal statistics and manageable backgrounds is achieved in the lepton+jets channels which have the signature of one isolated lepton, missing energy from one neutrino and four jets. The most precise measurements of top-quark properties are obtained from the lepton+jets channels.

Until recently, measurements of the top-quark mass $m_{\mathrm{t}}$ in the lepton+jets channels were limited by the statistical uncertainties. This is not the case for the analyses presented here, that use data sets of up to $320 \mathrm{pb}^{-1}$. It is therefore important to understand the systematic uncertainties in great detail and to minimize their impact on the precision of the top-quark mass. It turns out that in most cases the dominating source of systematic uncertainties is the jet energy scale. In determining the jet energy scale, the experiments have so far relied on detector calibration with test-beam data and reference processes, such as $E_{T}$ balancing in $\gamma+$ jets and dijet events. However, $t \bar{t}$ events in the lepton+jets channels provide an excellent tool to determine the jet energy scale in-situ: since the mass of the $\mathrm{W}$ boson is known with high accuracy, the overall energy scale for light jets can be determined by constraining the invariant mass of the two jets assigned to the hadronic decays of the $\mathrm{W}$ to the $\mathrm{W}$ mass. Two of the six analyses presented here make use of the in-situ calibration. Not surprisingly, these analyses lead to the most precise measurements of the top-quark mass.

As a novelty measurement, one analysis is presented that derives the top-quark mass solely from tracking information and hence minimizes the impact of jet energy scale uncertainties. The currently large statistical error of this method will be less problematic when data sets larger than $1 \mathrm{fb}^{-1}$ are available in the near future.

\section{Measuring the top-quark mass in the lepton+jets channel}

The CDF and DØ collaborations have applied several techniques in order to measure the mass of the top quark $m_{\mathrm{t}}$. The methods can be divided into two main categories: a) template methods, where a quantity strongly correlated to the top-quark mass is calculated for each event and then templates of the distribution for several top-quark mass hypotheses are fitted to the distribution of all data events to obtain the most likely top-quark mass, and b) matrix element and dynamic likelihood methods, where for each event a probability is calculated to originate from a $\bar{t} \bar{t}$ events with a hypothetical top-quark mass value. The probabilities of all events are combined into a final likelihood, which is then maximized as a function of $m_{\mathrm{t}}$.

The steps common to all analyses are briefly described below, followed by a more detailed description of three CDF and three DØ measurements. First of all, events are selected that are signal-like, i.e., they contain an isolated lepton, at least four jets and substantial missing transverse 
energy. After this step, the only major backgrounds in the data sample are $\mathrm{W}+\mathrm{jets}$ and QCD events. Additional cuts or b-tagging are used to reduce these backgrounds. In the third step, the final state is reconstructed, assigning the isolated lepton and each of the jets to one of the two top quarks. Finally, the last step is then to extract the top-quark mass, e.g., from the invariant masses $m_{\mathrm{jj}}, m_{\ell v \mathrm{~b}}$ or combined likelihood curves as a function of $m_{\mathrm{t}}$.

\subsection{CDF Template method}

Events are selected if they have an electron or muon with $E_{T}\left(p_{T}\right)>20 \mathrm{GeV}$, missing transverse energy $>20 \mathrm{GeV}$, and at least four jets with $E_{T}$ requirements detailed below. The 165 lepton+jets candidates obtained after this preselection are further divided into four subsamples with different requirements on the number of b-tagged jets and minimum $p_{T}$ values of the jets according to Table 1. In this way, it is possible to account for different sensitivities and backgrounds in each subsample and therefore to enhance the statistical power of the overall analysis.

Table 1: Jet $E_{T}$ and $p_{T}$ cuts for the four subsamples.

\begin{tabular}{lllll}
\hline Category & 2-tag & 1-tag(T) & 1-tag(L) & 0-tag \\
\hline Jet $E_{T} \mathrm{j} 1-\mathrm{j} 3$ & $E_{T}>15$ & $E_{T}>15$ & $E_{T}>15$ & $E_{T}>21$ \\
cuts $(\mathrm{GeV}) \mathrm{j} 4$ & $E_{T}>8$ & $E_{T}>15$ & $15>E_{T}>8$ & $E_{T}>21$ \\
b-tagging & 2 tags & $1 \mathrm{tag}$ & $1 \mathrm{tag}$ & 0 tag \\
No. events: & 25 & 63 & 33 & 44 \\
No. events with & 16 & 57 & 25 & 40 \\
$\chi^{2}<9$ & & & & \\
\hline
\end{tabular}

For each event, a kinematic $\chi^{2}$ fit is performed for each possible combination of assigning the four jets with the original partons and the two solutions for the neutrino $p_{z}$ component. Note that the $p_{z}$ component cannot be measured in the detector, but instead it can be calculated from the constraint $m_{\ell v}=m_{W}$. The kinematic $\chi^{2}$ to minimize is defined by:

$$
\begin{aligned}
\chi^{2} & =\sum_{i=\ell, 4 \mathrm{jets}} \frac{\left(\hat{p}_{T}^{i}-p_{T}^{i}\right)^{2}}{\sigma_{i}^{2}}+\sum_{j=x, y} \frac{\left(\hat{p}_{j}^{U E}-p_{j}^{U E}\right)^{2}}{\sigma_{j}^{2}} \\
& +\frac{\left(m_{j j}-m_{W}\right)^{2}}{\Gamma_{W}^{2}}+\frac{\left(m_{\ell v}-m_{W}\right)^{2}}{\Gamma_{W}^{2}}+\frac{\left(m_{b j j}-m_{t}^{\text {reco }}\right)^{2}}{\Gamma_{t}^{2}}+\frac{\left(m_{b \ell v}-m_{t}^{\text {reco }}\right)^{2}}{\Gamma_{t}^{2}}
\end{aligned}
$$

where the first sum runs over the lepton and the four leading jets and the second sum runs over the unclustered energy. The mass of $\mathrm{t}$ and $\overline{\mathrm{t}}$ must have the same value, and the two $\mathrm{W}$ masses are constrained to the PDF value of $m_{\mathrm{W}}$. The latter constraint allows for an in-situ calibration of the jet energies: In the final template fit, both $m_{\mathrm{t}}$ and the overall jet energy scale (JES) factor are free parameters. In the untagged subsample, there are 12 possible jet-parton combinations, 6 in the 1-tag subsamples and 2 in the 2-tag subsample. In either case, there are 2 choices for the neutrino four-vector. Only the combination with the smallest $\chi^{2}$ is considered, and only if $\chi^{2}<9$.

The distribution of the reconstructed masses $m_{\mathrm{jjb}}, m_{\ell v \mathrm{~b}}$, and $m_{\mathrm{jj}}$ in the selected signal Monte Carlo events are fitted with analytical functions to obtain smooth template distributions, Figure 1.

Mass templates for the $\mathrm{W}+$ jets background are obtained in a similar way to the signal templates. 


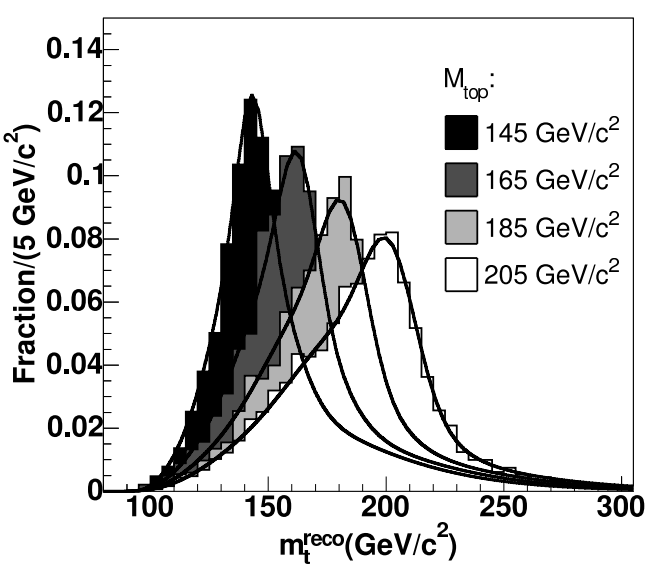

Figure 1: Signal $m_{\mathrm{t}}$ templates for various topquark masses.

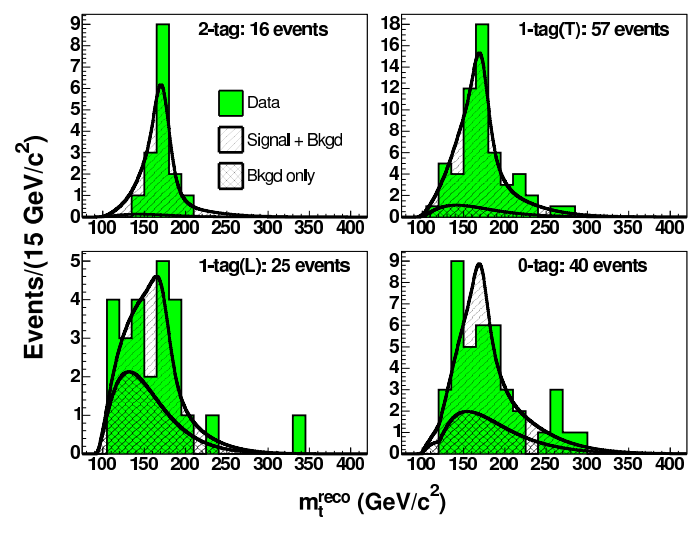

Figure 2: Reconstructed top-quark mass distribution in data for the four subsamples.

The best value of top-quark mass and the energy scale is finally extracted by comparing the signal and background templates to the reconstructed distributions in data using an unbinned likelihood fit. The likelihood definition contains terms for the true top-quark pole mass, jet energy scale, and the expected number of signal and background events. For each subsample it is given by:

$$
\mathscr{L}_{\text {sample }}=\mathscr{L}_{\text {shape }}^{m_{\mathrm{t}}^{\text {reco }}} \times \mathscr{L}_{\text {shape }}^{m_{j j}} \times \mathscr{L}_{\text {nev }} \times \mathscr{L}_{\text {bg }} .
$$

The detailed description of these terms can be found in [4].

The total likelihood is given by the product of the likelihoods for all four subsamples and a term for the overall JES constraint:

$$
\mathscr{L}_{\text {total }}\left(m_{\mathrm{t}}, J E S\right)=\mathscr{L}_{2 \text {-tag }} \times \mathscr{L}_{1-\operatorname{tag}(\mathrm{T})} \times \mathscr{L}_{1-\operatorname{tag}(\mathrm{L})} \times \mathscr{L}_{0 \text {-tag }} \times \mathscr{L}_{\text {JES }}
$$

The likelihood has a total of ten free parameters (number of signal and background events in four subsamples, JES, and $m_{\mathrm{t}}$ ). Fits to reconstructed masses in data are shown in Figure 2.

The fit yields a top-quark mass of $m_{\mathrm{t}}=173.5_{-3.6}^{+3.7}($ stat. $+J E S)$. The statistical uncertainty from the jet energy scale only is $\pm 3 \mathrm{GeV}$. The total systematic uncertainties add to $1.3 \mathrm{GeV}$ where the largest contribution comes from the b-jet energy scale and final state radiation.

\subsection{CDF Dynamic Likelihood method}

The "Dynamic Likelihood Method" calculates a per-event likelihood for the event to be a t $\bar{t} \rightarrow \ell+$ jets event. The likelihood is based on a matrix element calculation of the differential t $\bar{t}$ cross section and on transfer functions that describe the jet energy resolution in the transition from partons to jets:

$$
L^{i}\left(m_{\mathrm{t}}\right)=\int \sum_{\text {jet comb }} \sum_{v \text { sol. }} \frac{2 \pi^{4}}{\text { flux }}\left|M^{2}\right| F\left(z_{1}, z_{2}\right) f\left(p_{T}\right) w\left(x, y_{t} ; m_{\mathrm{t}}\right) d x
$$




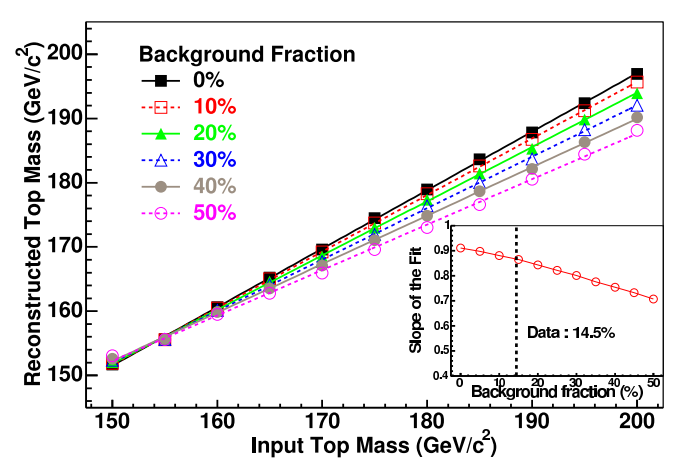

Figure 3: Extracted top-quark mass as a function of the generated mass for various background fractions between $0 \%$ and $50 \%$.

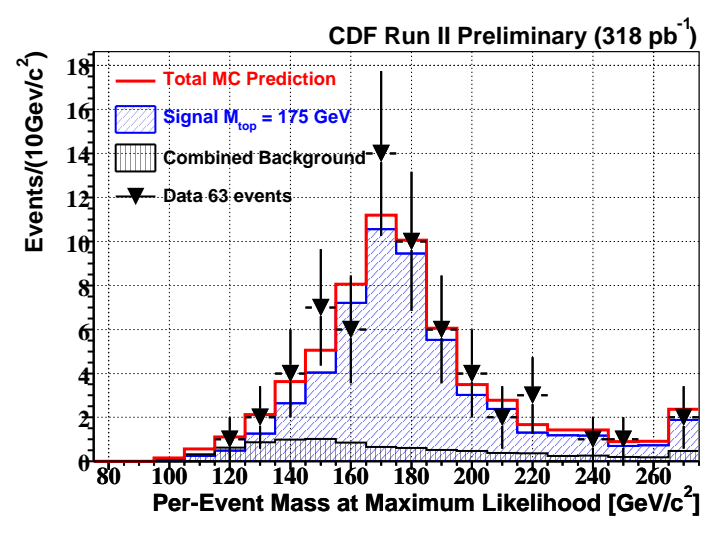

Figure 4: Mass distribution for the combination with maximal likelihood.

where $M$ is the leading-order matrix element for the $\mathrm{t} \overline{\mathrm{t}}$ process, $F\left(z_{1}, z_{2}\right)$ are parton distribution functions, $f\left(p_{T}\right)$ describes the $p_{T}$ distribution of the $\mathrm{t} \overline{\mathrm{t}}$ system, and $w\left(x, y ; m_{\mathrm{t}}\right)$ are the transfer functions (detector resolution). As in the case of the template method, one has to sum over all possible jet-parton combinations and the two solutions for the neutrino momentum. A final likelihood is obtained by the product of all event likelihoods: $L=\prod_{\text {events }} L^{i}$.

The analysis is based on $318 \mathrm{pb}^{-1}$ and requires exactly four jets with $E_{T}>15 \mathrm{GeV}$. The restriction to four jets is made to minimize the contamination from initial and final state radiation jets, since only a leading-order matrix element calculation is utilized.

The likelihood definition in Equation (2.1) does not contain a term for the background probability, instead the background is reduced by requiring at least one of the four jets to be b-tagged by a secondary-vertex algorithm. This requirement also reduces the possible jet-parton combinations.

A calibration curve, relating the extracted top mass after minimizing $-\log (L)$ to the input top mass, is obtained from a set of Monte Carlo pseudo-experiments (Figure 3). The non-unit slope is caused by the dependence of the transfer functions on the top-quark mass and by the presence of background. The background consists mainly of W plus heavy flavor jets and QCD (non-W) events and is estimated to be $9.2 \pm 1.8$ events. The total likelihood is finally computed in data from 63 candidate events and yields a top-quark mass of $m_{\mathrm{t}}=173.2_{-2.4}^{+2.6}($ stat. $) \pm 3.2($ syst. $) \mathrm{GeV}$. The systematic uncertainties are dominated by jet energy scale corrections $\left(\Delta m_{\mathrm{t}}=3.0 \mathrm{GeV}\right)$.

As a cross check for the data/MC agreement, the reconstructed mass for the combination with the largest likelihood in each event is depicted in Figure 4 for a generated signal mass of $m_{\mathrm{t}}=175 \mathrm{GeV}$.

\subsection{CDF Decay Length Method}

A new method that minimizes the impact of uncertainties from the jet energy scale is the "decay length technique". It relies on tracking rather than calorimetry to measure the top-quark mass. Since at the Tevatron top-quark pairs are produced almost at rest, the boost of b-quarks in the top decays can be written as

$$
\gamma_{b}=\frac{m_{t}^{2}+m_{b}^{2}-m_{W}^{2}}{2 m_{t} m_{b}} \approx 0.4 \frac{m_{t}}{m_{b}}
$$




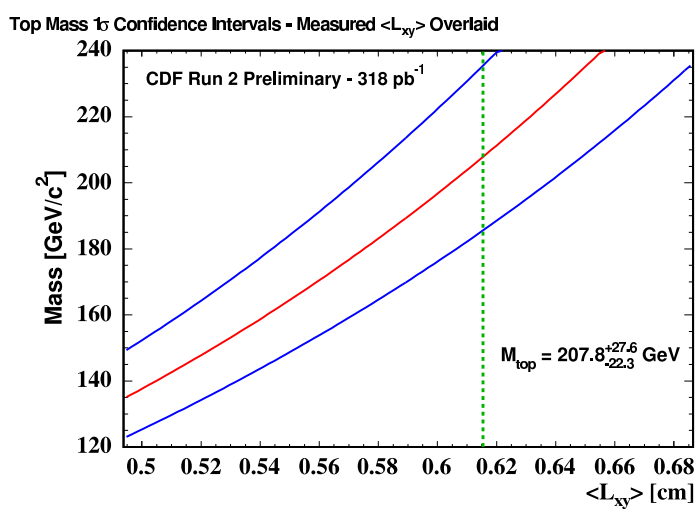

Figure 5: Calibration curve for the top-quarks mass as a function of the average decay length $L_{x y}$.

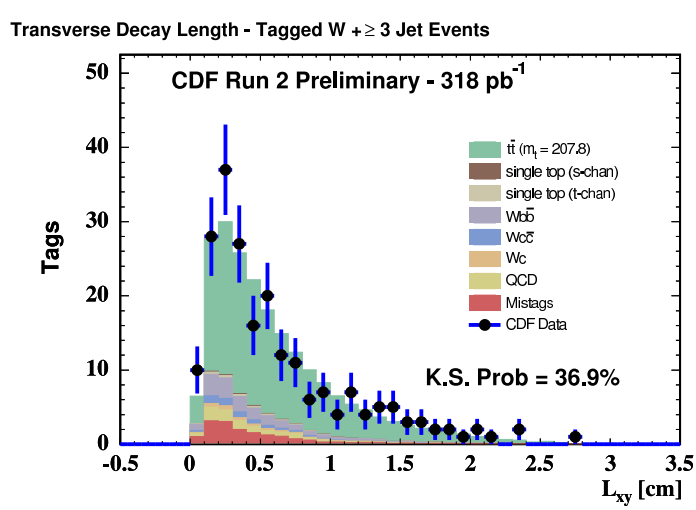

Figure 6: $L_{x y}$ distribution in data and signal and background simulation. The Monte Carlo distributions are normalized to the observed data.

The boost, in turn, is directly correlated with the decay length of b-hadrons, and a measurement of the average decay length $\left\langle L_{x y}\right\rangle$ thus gives access to $m_{\mathrm{t}}$.

The analysis is based on $318 \mathrm{pb}^{-1}$ of data and requires an electron (muon) with $E_{T}>20 \mathrm{GeV}$ $\left(p_{T}>20 \mathrm{GeV}\right)$, missing transverse energy $>20 \mathrm{GeV}$ and at least three jets with $E_{T}>15 \mathrm{GeV}$. Additionally, at least one of the jets being b-tagged with an algorithm that looks for secondary vertices. A calibration curve of the top-quark mass as a function of the mean decay length is obtained from Monte Carlo simulations of the signal and the expected background (Figure 5). Finally, the event selection is applied to data, and $\left\langle L_{x y}\right\rangle$ is measured in $216 \mathrm{t} \overline{\mathrm{t}}$ candidate events. The data distribution is compared to the signal and background prediction in Figure 6.

The extracted top-quark mass is $m_{\mathrm{t}}=207.8_{-22.3}^{+27.6}$ (stat.) \pm 6.5 (syst.) GeV. The largest contributions to the total systematic uncertainty stems from the background shape and normalization, which accounts for a $3.3 \mathrm{GeV}$ uncertainty, and the limited Monte Carlo statistics used to derive the man $L_{x y}$ data/MC ration, which accounts for a $5.1 \mathrm{GeV}$ uncertainty.

\subsection{DØ Template method}

The DØ template method is based on the same concept as the corresponding CDF analysis: the mass distribution obtained from a kinematic fit on collider data is compared to mass templates from simulated events with known top-quark masses, though the details of the event selection and the fitting procedure are different.

Events are selected if they have at least one isolated electron (muon) with $p_{T}>20 \mathrm{GeV}$, missing transverse energy greater than $20 \mathrm{GeV}$, and at least four jets with $p_{T}>15 \mathrm{GeV}$, out of which the three leading jets must satisfy $p_{T}>20 \mathrm{GeV}$. A kinematic fit is performed for each possible jet-parton combination, and only the one with lowest $\chi^{2}$ is considered (where the $\chi^{2}$ must be less than 10).

After the preselection, the main background is comprised of $\mathrm{W}+$ jets events. To enhance the $t \overline{\mathrm{t}}$ signal fraction, further discrimination between signal and background is achieved by constructing a discriminant that has little sensitivity to the actual value of the top-quark mass. The low-bias

discriminant $D_{L B}$ is a combination of four topological variables and is shown in Figure 7. Events 


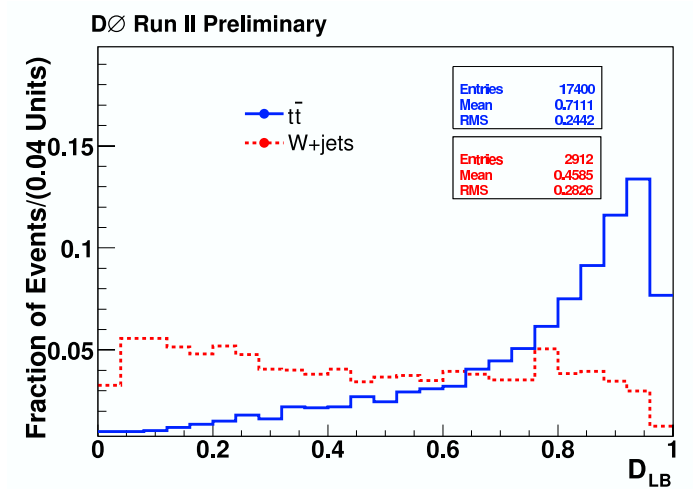

Figure 7: The low-bias discriminant.

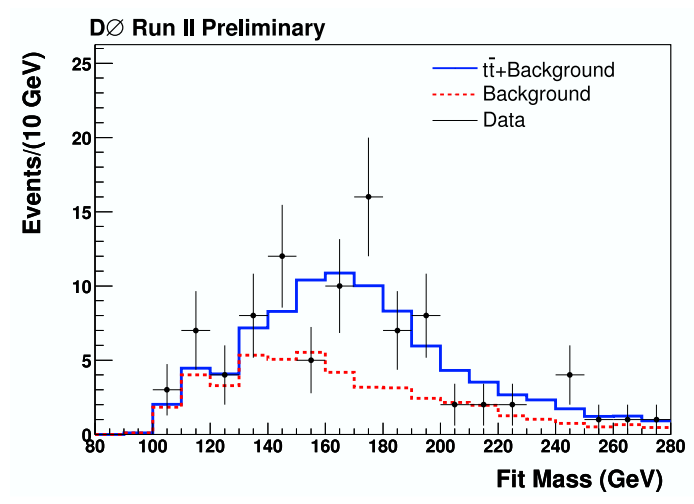

Figure 9: Fit mass distribution in the untagged sample in the template method.

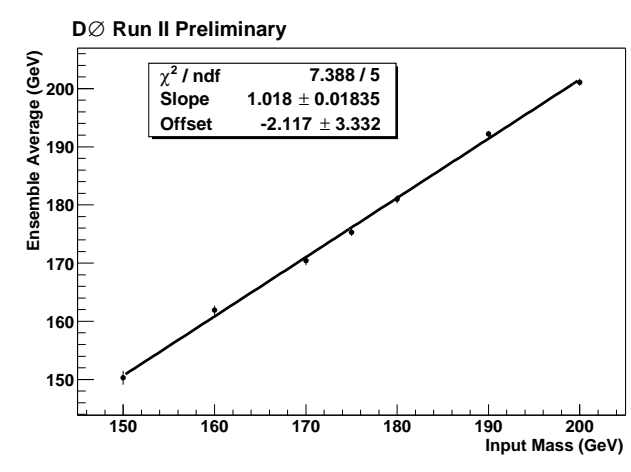

Figure 8: Calibration curve for the template method

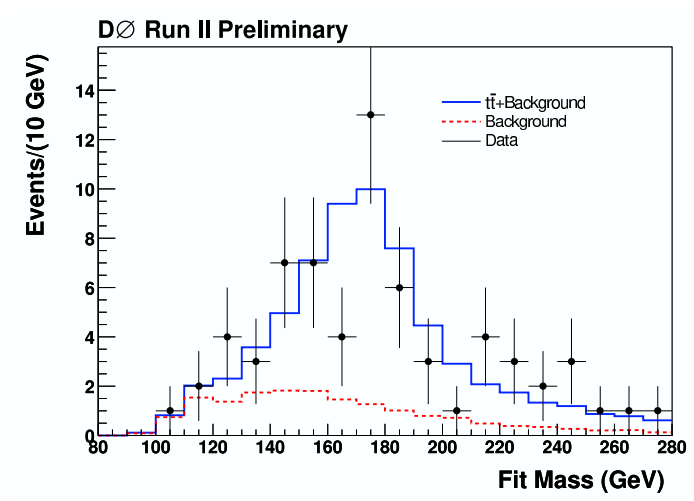

Figure 10: Fit mass distribution in the b-tagged sample in the template method.

pass the selection if $\mathrm{D}_{\mathrm{LB}}>0.4$. 94 candidates remain in the data with an estimated signal-tobackground ratio of 1:1. To extract the top-quark mass, Monte Carlo template mass distributions for various values of $m_{\mathrm{t}}$ are compared to the data using a binned likelihood fit. The performance of this method is studied in ensembles of simulated events (Figure 8). Within uncertainties, the calibration curve has a slope of one and an offset that is consistent with zero.

Figure 9 shows the fit mass distribution in data and in signal and background Monte Carlo. The signal and background distributions are shown in the fraction obtained from the fit. The preferred top-quark mass, extracted from the minimization of the likelihood, is $m_{\mathrm{t}}=169.9 \pm$ $5.8(\text { stat. })_{-7.1}^{+7.8}($ syst. $) \mathrm{GeV}$.

The template method was also performed using b-tagging to reduce the background and jetparton combinations. At least one jet is required to be tagged by a secondary-vertex algorithm, while no cut on the low-bias discriminant is applied. The selection yields $69 \mathrm{t} \overline{\mathrm{t}}$ candidates in data with an enhanced signal-to-background ratio of 3:1. The reconstructed fit mass for the b-tagged samples, which is shown in Figure 10, reveals a much more pronounced signal peak. The extracted top-quark mass is $m_{\mathrm{t}}=170.6 \pm 4.2$ (stat.) \pm 6.0 (syst.) GeV.

In both the topological and the b-tagged approach, the main systematic uncertainty arises from the jet energy scale $\left(\Delta m_{\mathrm{t}} \approx 6 \mathrm{GeV}\right)$. 

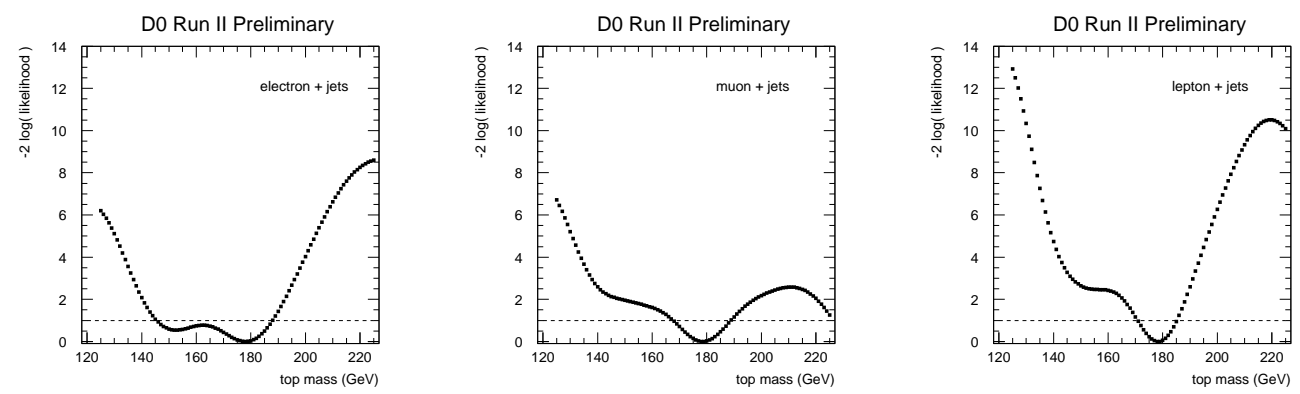

Figure 11: Mass likelihood curve for observed $t \bar{t}$ candidates in the e+jets channel (left), $\mu+j e t s$ channel (middle) and the combined $\ell+$ jets channel (right) in the ideogram method.

\subsection{DØ Ideogram Method}

The ideogram method is based on the same kinematic fit and makes use of the same low-bias discriminant as the template method. However, the Ideogram method includes all possible parton-jet combinations and neutrino solutions in the constrained kinematic fit to calculate an event likelihood, and there is no cut on the low-bias discriminant $\mathrm{D}_{\mathrm{LB}}$. Instead, $\mathrm{D}_{\mathrm{LB}}$ is used to derive the probability for an event to be background. The likelihood for the selected data sample is defined by

$$
\begin{aligned}
L\left(m_{\mathrm{t}}, P_{\text {sample }}\right) & =\sum_{i} w_{i}\left(P_{\mathrm{evt}} \cdot S\left(m_{i}, \sigma_{i}, m_{\mathrm{t}}\right)+\left(1-P_{\mathrm{evt}}\right) \cdot B G\left(m_{i}\right)\right), \\
w_{i} & =\exp \left(-\chi_{i}^{2} / 2\right), \\
S\left(m_{i}, \sigma_{i}, m_{\mathrm{t}}\right) & =\int d m^{\prime} G\left(m^{\prime}, m_{i}, \sigma_{i}\right) \cdot B W\left(m^{\prime}, m_{\mathrm{t}}, \Gamma_{\mathrm{top}}\right) .
\end{aligned}
$$

The sum in the first line runs over all 24 possible combinations, and each combination is assigned a weight $w_{i}$ according to the goodness of the kinematic fit. The signal likelihood shape $S$ is the convolution of a Breit-Wigner shape with Gaussian resolution functions describing the detector resolution, and $B G$ is the background shape as expected from $\mathrm{W}+\mathrm{jets}$ and QCD Monte Carlo simulations. The event purity $P_{\text {evt }}$ is a parameterized function of the discriminant $\mathrm{D}_{\mathrm{LB}}$.

After deriving a calibration curve from Monte Carlo ensemble tests, the method was applied to 191 candidate events selected in $160 \mathrm{pb}^{-1}$ of data. The likelihood curves are displayed in Figure 11. After corrections, the fitted top-quark mass is $m_{\mathrm{t}}=177.5 \pm 5.8$ (stat.) \pm 7.1 (syst.) GeV. The two largest contributions to the systematic uncertainties come from the jet energy scale $(4.8 \mathrm{GeV})$ and from signal modeling $(3.8 \mathrm{GeV})$.

\subsection{DØ Matrix Element Method}

The matrix element (ME) method was developed within the $\mathrm{D} \varnothing$ collaboration and yields the most precise single measurement of the top-quark mass from Run I data [2]. The same technique has also been applied to $320 \mathrm{pb}^{-1}$ of Run II data. This method makes maximal use of the event information by calculating a probability for an event to be signal or background from the matrix element for the $\mathrm{t} \overline{\mathrm{t}}$ signal process and the dominant background production ( $\mathrm{W}+\mathrm{jets}$ events). It is therefore similar 


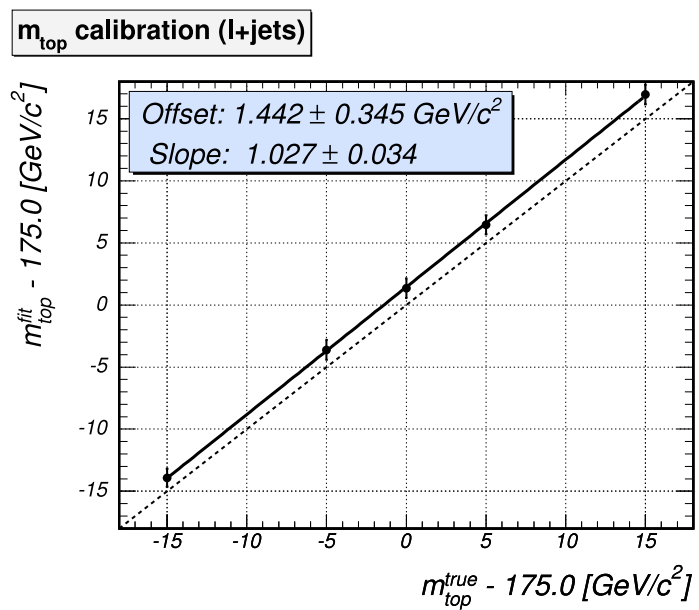

Figure 12: Calibration curve for $m_{\mathrm{t}}$ in the matrix element method.

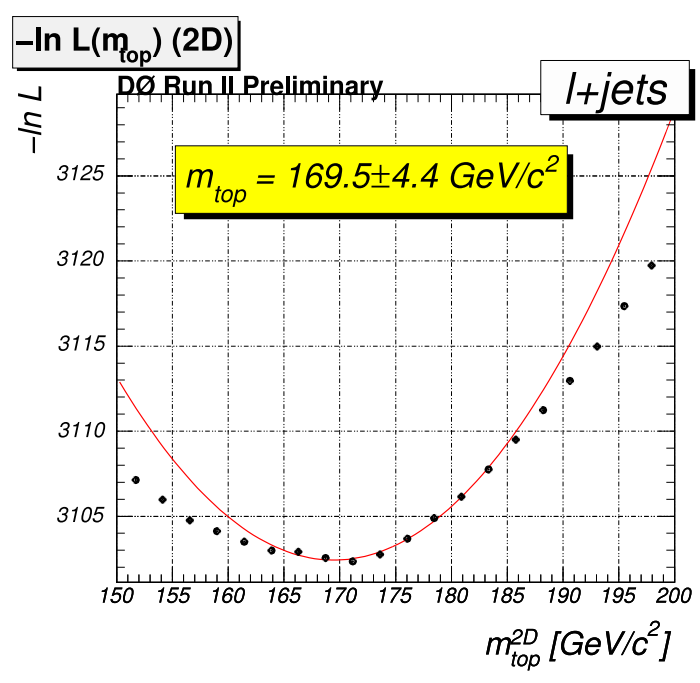

Figure 13: Projection of the two-dimensional likelihood curve to the $m_{\mathrm{t}}$ parameter.

in concept to the DLM method employed by CDF, but additionally takes into account a background term for the event probability. However, there is no b-tagging requirement.

In order to be less dependent on external jet energy scale corrections, the ME method fits both the top-quark mass and an overall JES factor. The expression for the signal probability is similar to Equation (2.1), and the event probability is given by

$$
P_{\mathrm{evt}}\left(x ; m_{\mathrm{t}}, J E S\right)=f_{\mathrm{top}} \cdot P_{\mathrm{sgn}}\left(x ; m_{\mathrm{t}}, J E S\right)+\left(1-f_{\mathrm{top}}\right) \cdot P_{\mathrm{bkg}}(x ; J E S)
$$

where $f_{\text {top }}$ is the fraction of $\bar{t} \bar{t}$ signal events in the selected data set, and $P_{\text {sgn }}$ and $P_{\text {bkg }}$ are the signal and background probabilities based on leading order matrix element calculations for the $\bar{t} \bar{t}$ and $\mathrm{W}+$ jets processes. The sensitivity to the jet energy scale enters through the jets from the hadronically decaying $\mathrm{W}$ boson, whose invariant mass is constrained to $m_{\mathrm{W}}$ in the $\mathrm{t} \overline{\mathrm{t}}$ matrix element. The total sample likelihood is defined by

$$
-\ln L\left(x_{1}, \ldots, x_{n} ; m_{\mathrm{t}}, J E S\right)=-\sum_{i=1}^{n} \ln \left(P_{\mathrm{evt}}\left(x_{i} ; m_{\mathrm{t}}, J E S\right)\right)
$$

and is minimized as a function of $m_{\mathrm{t}}, \mathrm{JES}$, and $f_{\mathrm{top}}$.

The method is calibrated using ensembles of simulated t $\bar{t}$ signal and $\mathrm{W}+$ jets background samples that correspond in size to the number of selected candidates in data (150 events). The calibration curve (Figure 12) has a small offset of $1.44 \mathrm{GeV}$ and a slope that is compatible with unity. After applying this method to the data, the minimum in the $-\log L$ curve is found to be $m_{\mathrm{t}}=169.5 \pm 4.4(\text { stat } .+J E S)_{-1.6}^{+1.7}($ syst . $) \mathrm{GeV}$. The relative JES scale factor is $1.034 \pm 0.034$, indicating that the light flavor jets are well calibrated. The fitted top fraction is $31.6_{-5.5}^{+4.9} \%$ which is consistent with the expectation from an independent measurement [3]. Once again, uncertainties from the jet energy scale contribute the most to the total systematic uncertainties on $m_{\mathrm{t}}$, along with $\mathrm{b}$ fragmentation and QCD contamination. 
Table 2: Summary of top-quark measurements.

\begin{tabular}{lllll}
\hline Method & $\begin{array}{l}\int \mathscr{L} d t \\
\left(\mathrm{pb}^{-1}\right)\end{array}$ & $\begin{array}{l}m_{\mathrm{t}} \\
(\mathrm{GeV})\end{array}$ & $\begin{array}{l}\Delta m_{\mathrm{t}} \text { (stat.) } \\
(\mathrm{GeV})\end{array}$ & $\begin{array}{l}\Delta m_{\mathrm{t}} \text { (syst.) } \\
(\mathrm{GeV})\end{array}$ \\
\hline CDF Template & 318 & 173.5 & ${ }_{-3.6}^{+3.7}$ & 1.3 \\
CDF DLM & 318 & 173.2 & ${ }_{-2.4}^{+2.6}$ & 3.2 \\
CDF Decay length & 318 & 207.8 & ${ }_{-22.3}^{+27.6}$ & 6.5 \\
DØ Template (topo) & 230 & 169.9 & ${ }_{-7.1}^{+7.8}$ & 5.8 \\
DØ Template (b-tag) & 230 & 170.6 & 4.2 & 6.0 \\
DØ Matrix Element & 320 & 169.5 & 4.4 & +1.7 \\
DØ Ideogram & 160 & 177.5 & 5.8 & 7.1 \\
\hline
\end{tabular}

\section{Summary}

A variety of analysis techniques have been applied to a large set of Tevatron Run II p $\bar{p}$ collision data in order to measure the mass of the top quark in lepton+jets final states with high precision. The results are summarized in Table 2 . The measurements are no longer limited by the statistical uncertainty. Uncertainties from the jet energy scale dominate the systematic uncertainties in most cases, and some analyses have applied in in-situ calibration of the jet energies using the $\mathrm{W}$ mass constraint of the two lights jets in the hadronic $\mathrm{W}$ decays. This approach improves the understanding of the energy scale for light jets.

Combining the results from both experiments in the dilepton, lepton+jets and all-jets channel, the new preliminary world average is [5]

$$
m_{\mathrm{t}}^{\text {world average }}=172.7 \pm 2.9 \mathrm{GeV} .
$$

Both the CDF and the DØ experiments plan to soon present new measurements based on an even larger dataset and with less systematic uncertainties. This will allow for additional precision tests of the Standard Model and will increase the sensitivity to new physics.

\section{References}

[1] http://www-cdf.fnal.gov/physics/new/top/top.html http://www-d0.fnal.gov/Run2Physics/top/top_public_web_pages/top_public.html

[2] V. M. Abazov et al. [D0 Collaboration], Nature 429, 638 (2004) [hep-ex/0406031]

[3] V. M. Abazov et al. [D0 Collaboration], Phys. Lett. B 626, 45 (2005)

[4] A. Abulencia et al. [CDF Collaboration], hep-ex/0510048, submitted to Phys. Rev. D

[5] [CDF and D0 Collaborations], arXiv:hep-ex/0507091 